\section{Revue critique de fixxion française \\ WX. contemporaine}

23 | 2021

Statut du personnage dans la fiction contemporaine

\title{
Robinson, Pierre, Mathilde et les autres - Cadiot et ses personnages
}

Propos recueillis par Nathalie Piégay et Dominique Rabaté

Olivier Cadiot, Nathalie Piégay et Dominique Rabaté

\section{OpenEdition}

\section{Journals}

Édition électronique

URL : https://journals.openedition.org/fixxion/752

DOI : 10.4000/fixxion.752

ISSN : 2295-9106

\section{Éditeur}

Ghent University

Référence électronique

Olivier Cadiot, Nathalie Piégay et Dominique Rabaté, « Robinson, Pierre, Mathilde et les autres - Cadiot et ses personnages », Revue critique de fixxion française contemporaine [En ligne], 23 | 2021, mis en ligne le 15 décembre 2021, consulté le 17 février 2022. URL : http://journals.openedition.org/fixxion/ 752 ; DOI : https://doi.org/10.4000/fixxion.752

Ce document a été généré automatiquement le 17 février 2022.

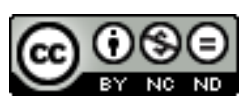

Les contenus de la Revue critique de fixxion française contemporaine sont mis à disposition selon les termes de la licence Creative Commons Attribution - Pas d'Utilisation Commerciale - Pas de Modification 4.0 International. 


\title{
Robinson, Pierre, Mathilde et les autres - Cadiot et ses personnages
}

Propos recueillis par Nathalie Piégay et Dominique Rabaté

\author{
Olivier Cadiot, Nathalie Piégay et Dominique Rabaté
}

\section{NOTE DE L'AUTEUR}

La transcription et l'édition ont été assurées par Nina Rocipon.

Paris, le 10 septembre 2021

DOMinique RABATÉ : Cher Olivier, nous sommes venus, Nathalie et moi, parler de la question du personnage dans tes livres, dans ton œuvre. Pour commencer, on peut dessiner une sorte de direction générale, puisque le propos de ce numéro est de réfléchir au statut du personnage aujourd'hui : faut-il parler de retour, ou non, ce mot de "retour » que tu as mis dans un de tes titres. On s'est dit que c'était intéressant d'en parler avec toi parce qu'on a l'impression que la question du personnage est à la fois centrale et problématique. D'une certaine manière à chaque fois tu la remets en jeu dans les livres que tu fais. Alors entrons dans le questionnement par une question un peu massive : qu'est-ce qui arrive au personnage de roman quand il devient plutôt une voix ?

Olivier Cadiot : Très bien.

D.R. : Il faut que je précise ! Car cette question a été pour moi le point de départ du travail autour de des Forêts, Beckett, de l'idée de littérature de l'épuisement. Je voulais montrer dans une histoire plus longue, qui englobe Proust aussi, ce qui se passe quand, au lieu d'avoir un personnage constitué, ça devient plutôt une sorte de présence vocale, mais polyphonique, diffuse, spectrale, ironique... On pourrait commencer comme ça : est-ce que pour toi le rapport ou le conflit entre voix (au pluriel aussi bien) et personnage(s) est essentiel... ? Comment le situerais-tu par rapport à ce déplacement qu'on peut schématiser de Proust à Beckett pour prendre deux auteurs qui sont importants pour toi ?

O.C. : Ce qui est curieux c'est que je pourrais presque faire une chronologie à l'envers, dans l'autre sens, et dire : tiens, c'est étrange, il n'y a de moins en moins de voix, dans la littérature d'aujourd'hui, il y a de moins en moins de voix, de polyphonie de sens et de formes et de plus en plus de personnages-narrateurs soi-disant réels. Donc on est 
revenu peut-être au XIX ${ }^{e}$. C'est intéressant. Mais de qui on parle ? C'est très difficile de savoir, car tous les modes d'écrire continuent parallèlement à exister même en disparaissant... et s'hybrident à l'infini. Ça complique la lecture. Pour simplifier on dira que le personnage est un problème à étudier, et pour moi aussi même si je ne me suis jamais posé la question en ces termes - je vis avec. Dans ma petite histoire à moi, j'ai l'impression qu'au départ je déniais la fonction personnage. En bon enfant de la modernité, je lui déniais le droit d'exister, parce que le personnage, pour moi, c'était la place de la psychologie. Comme dans Proust tous les personnages sont ridicules sauf une voix intérieure... Et donc naturellement, pour éviter de trop les développer, j'ai voulu centrer mes livres sur un seul héros, j'ai fait une série de solos polyphoniques. J'ai l'impression d'accepter de ne me poser la question du personnage vraiment, pour la première fois, que très récemment avec et à partir de Providence. Je fais peut-être une mue. Je dois être quand même dans une espèce de double discours. C'est paradoxal. D'un côté je pense qu'il n'y a pas de personnage, il n'y a que des voix : j'écris L'art poétic'; et de l'autre je me lance dans un solo qui va durer vingt-cinq ans. Avec le premier livre de cette série Futur, ancien, fugitif, je tiens mon Robinson - et je ne le lâche plus.

D.R. : Robinson, est-ce que pour toi c'est un personnage ou un anti-personnage ? Quand il apparait, quand tu le fabriques, comment ça se passe? Et pourquoi résiste-t-il et tient-il aussi longtemps?

NATHALIE PIÉgaY : Dans un entretien, tu disais que c'est "l'employé modèle pour un roman", ça veut dire quoi?

o.c. : C'est parfait! [rires] Enfin, je ne dis pas que ce que j'ai dit est parfait, je dis que ça répond un peu au problème, que la flèche est dans la cible.

N.P. : L'employé modèle, ça fait penser à La vie mode d'emploi aussi. Et c'est également un personnage de mythe.

o.c. : C'est ça. C'est l'employé modèle, c'est une couverture. Il me protège en me dédoublant. C'est lui qui prend les coups. Ce n'est pas que je me cache en lui comme dans un épouvantail ou dans un mannequin, c'est qu'il m'emmène partout. C'est un personnage prospectif. Il a de l'entrain même s'il n'est pas vraiment un héros, c'est Monsieur tout le monde. Un homme ordinaire, mais comme tour à tour augmenté, diminué, vendredisé. J'ai choisi le mythe le plus accessible : tout enfant se prend pour Robinson dans sa cabane. C'est une imagerie de bande dessinée. Ça fait une affiche simple, il suffit de mettre un type avec un perroquet ou un chapeau, on peut écrire dessus en cas d'extrême solitude: appelez le SoS Robinson. C'est un mythe raisonnable et à la portée de tous. Devenir le petit ingénieur, l'explorateur de sa vie. Ce qui est curieux c'est qu'en relisant des notes il y a très longtemps je me suis rendu compte que le personnage ne s'appelait pas d'abord Robinson. Il avait le prénom... de mon père.

D.R. : Le premier personnage auquel tu penses portait le prénom de ton père?

O.C. : Oui. Futur, ancien, fugitif, au tout départ, avant même que ce soit un livre qui fonctionne, comme une sorte d'encyclopédie en boucle, n'était qu'une espèce de description d'une vie... de mon père dans son enfance. J'ai eu un père assez dur, assez complexe, très intéressant, mais violent à bien des égards. Donc je voulais peut-être le réhabiliter en le décrivant dans son enfance... enfin... de manière un peu naïve, j'essayais de retrouver le bon sujet en lui. 
N.P. : Il y avait donc une sorte de portrait de personnage dans ce qui deviendra Futur, ancien, fugitif?

O.C. : À ce moment de ma vie je fais le vide, je fabrique du vide, c'est ma seule option dans le monde dans lequel je vis à ce moment-là, et sur le vide de la page je fais intensément du cut-up au début des années 80 . Je fais mon premier livre avec cette méthode. Mais dès le deuxième livre, je convertis cette technique en une sorte de roman, ce sera Futur, ancien, fugitif. Comme s'il fallait s'échapper du minimaliste émotif. Pour découper la vie en structure.

D.R. : Continuons un peu en spirale, avec des retours. Robinson, c'est clairement celui qui repart à zéro... C'est la lecture qu'en propose Joyce, il dit que c'est le héros anglais absolument - car il déteste Robinson qui est l'essence du capitalisme...

o.C. : C'est ce que dit Deleuze, c'est un petit banquier protestant.

D.R. : Exactement, on le met tout seul sur une île...

O.C. : C'est atroce, le texte de Defoe est atroce, je déteste ce texte. [rires]

D.R. : C'est un Robinson qui est très Vendredi quand même.

O.C. : C'est un Robinson qui est très Vendredi ! Alors voilà, on commence à rentrer dans la complexité du truc.

D.R. : Mais ils sont deux...?

O.C.: Bien entendu ils sont deux, mais je les ai fusionnés. Et après je me suis beaucoup servi du fait que Robinson devenait Vendredi, qu'il était vraiment celui qui ne sait pas parler au départ de l'histoire, qui n'a aucune maîtrise. Donc j'ai fait un anti-Robinson, en prenant Vendredi.

N.P. : Il y a aussi un principe d'accélération, comme si le fait que ça aille plus vite, comme dans le burlesque, les cartoons, ça coupe court à la psychologie, ça donne du rythme au corps, mais ça ne laisse pas tellement place non plus à la hiérarchie personnage principal, personnage secondaire. Ça ne peut pas s'empiler comme ça, ça part dans un rythme...

o.C. : Oui, c'est le comique qui accélère, la torsion, parce que le comique tord le problème, il y a quelque chose de tordu...

N.P. : Il tord aussi les corps.

O.C. : Il tord tout. Le comique va plus vite. C'est grâce à ce trait rapide qu'il y a apparition d'une voix et d'une présence. Ça fait personnage.

D.R. : Puisqu'on évoque le cut-up, il me semble que Médecine générale produit un effet de boucle très impressionnant, avec les poèmes de la fin du livre.

o.c. : Bien sûr qu'il y a brièvement du cut-up à la fin, c'est le poème involontaire de mon père que j'ai trouvé en marge d'un manuscrit où il tentait de raconter sa vie. Une série de remarques sur les arbres et les voix disparues - C'est à pleurer de beauté.

D.R. : Avant d'en venir à Médecine générale, encore un mot de Robinson, qui est le seul et premier mythe moderne. Et ce qui me frappe, quand je te lis : ton Robinson, c'est quand même celui qui sort d'un naufrage, c'est l'endeuillé.

o.c. : C'est quand même l'endeuillé, absolument.

D.R. : Donc la vitesse, le comique s'élèvent sur fond beaucoup plus sombre.

O.C. : L'auteur, le narrateur, l'île est entièrement endeuillée, tout est endeuillé de A à Z. Mais ça finit par fonctionner. Il y a comme un livre parallèle, un livre de joie pure. 
Ça fait un ruban de Möbius. L'espace du livre est traversé en tout sens et à toute vitesse par le personnage.

D.R. : Sa vitesse de projection?

O.C.: Sa vitesse de projection. C'est une machine, c'est une sublime machine humaine. Pour en dire plus sur notre personnage on pourrait dire, pour rire, qu'il serait comme un malade... structuraliste. Il y a eu un naufrage, et puis après on ouvre des coffres. Qu'est-ce que Robinson trouve au bord de la plage? Des caisses abandonnées remplies d'outils et d'objets. Un résumé de civilisation. Mais pratique, il suffit de réassembler ses objets pour avoir une vie mode d'emploi. Un cut-up de son pays d'origine. Et avec ça, ce gars, au lieu de rester à la plage tranquille et de rester en vacances forever, reconstruit un monde entier. Il ne sait pas s'arrêter. Au lieu de faire des petits poèmes il se lance dans de gros romans inachevés. Serait-ce une définition de notre personnage : quelqu'un qui contient des mondes à bâtir ? J'envoie un personnage principal dans une mission. Les personnages secondaires le restent vraiment, ils ont besoin d'être comme dans Alice au pays des merveilles au fond: des cartes. Ils n'ont pas d'épaisseur. Alors que le héros est une chambre d'écho. On va arriver à la voix: là, curieusement, personnage et voix sont confondus complètement. Je ne cesse de faire des livres hybrides. Futur, ancien, fugitif, par exemple, est un roman par listes.

N.P. : Ces listes gardent un rapport avec la poésie... II me semble qu'elles créent deux rapports différents au temps: il y a la liste, ce que Dominique disait, de l'endeuillé, qui récolte ce qu'il y avait avant, pour ne pas perdre; et puis il y a la liste prospective, qui regarde devant... je trouve qu'il y a les deux aspects dans ce rapport à la liste. Soit d'abord, une fonction conservatoire, on recueille, ce qu'on a peur d'oublier aussi.

o.c. : C'est ça, donc on recueille des voix-corps.

N.P. : On recueille des voix, des mots, des fonctions... mais il y a aussi une liste qui est programmatique, la liste des choses à faire.

D.R. : La to-do list...

N.P. : Donc je trouve que c'est une façon de déjouer la mélancolie de l'endeuillé, ces listes...

O.C.: Elles sont prospectives. C'est ce qu'on appelait autrefois des embrayeurs. L'embrayeur idéal, c'est “maintenant ». Donc ce n'est pas que prospectif, c'est aussi occuper le présent, ce qui revient au même. Ce n'est pas tout à fait "quand je serai grand, je ferai ça", c'est : "tout de suite"... "tout de suite je vais construire un bassin", "tout de suite je vais faire ça”... Quand dire, c'est faire. D'ailleurs Futur, ancien, fugitif, c'est la description des trois temps du moteur à combustion. C'est un moteur à trois temps, c'est une valse à trois temps, Futur, ancien, fugitif: qu'est-ce que c'est que conjuguer au futur, conjuguer à l'ancien et conjuguer au fugitif, c'est-à-dire justement être dans le fuyant...

D.R. : Je pensais : embrayer... le personnage Robinson, dont on parle depuis le début, c'est aussi un opérateur. Embrayeur, opérateur: effectivement il a cette fonction. Mais il a un petit quelque chose aussi, je reviens à l'idée qu'il y aurait quand même une sorte de personnage poétique, c'est-à-dire très silhouetté... moi il me fait aussi penser à Plume.

o.c. : C'est vrai.

D.R. : Comme Plume d'ailleurs, ou comme les personnages de Michaux, il se bat contre des projections de figures paternelles oppressantes, il les massacre et puis elles reviennent. Ce n'est pas un personnage de roman. Parce que si le mode c'est le fugitif, il est tout le temps dans des présents très ponctuels, hyperactifs... Comment arrive-t-on dans ce cas-là à 
construire un roman? Dans le premier livre, ce que tu fais, c'est ce que tu appelles le "kit" ? C'est-à-dire les éléments et leur plan de montage possible?

o.c. : C'est ça, exactement.

D.R. : Mais alors après, comment ça se passe? Pour que Robinson aille dans le monde, sorte de l'île?

O.C.: Bien sûr, deuxième tome, Le colonel des zouaves. Butler's literature et roman d'espionnage, puisque ça se termine dans une espèce de faux polar. C'est une prose, ce n'est plus un travail par fragments. Du coup ce qui m'a intéressé, c'est sa souplesse, sa ductilité, son hyperémotivité, ses qualités réceptrices, son endurance... Parce qu'il est toujours obligé, à chaque fois, de faire du sport. Parfois c'est du yoga mental comme il le dit dans Un nid pour quoi faire. Chaque fois il change d'exercice : les courses à pied dans Le colonel. Elles sont poétiques et peuvent être récitées à toute pompe rythmiquement. J'aurais pu, si j'avais continué à faire ce que je faisais avant, extraire, et publier telles quelles ses courses et en faire des sortes de chants poétiques, cela ne m'était plus possible. La poésie est inadmissible, on le sait bien: j'avais besoin de protéger ce flot charnel dans un flux de prose, encore une fois j'avais besoin d'une couverture. J'avais besoin de faire une sorte de coque, d'écrin de prose pour faire passer ça.

N.P. : Ça veut dire que la forme-roman, c'est un contenant?

o.C. : C'est un contenant... c'est surtout un transmetteur, c'est conducteur, comme dirait David Lapoujade à propos de James, c'est un personnage conducteur, c'est très beau.

N.P. : C'est moins "ça contient" que "ça conduit"...

o.c. : Ça conduit, ça revient à ce que tu disais, c'est toujours le présent de la prospective, tout à fait d'accord. Donc c'est un ingénieur aussi, et pas vraiment un écrivain. Et un artiste raté.

N.P. : Ce qui est intéressant par rapport à ce que suggérait Dominique avec Plume, c'est qu'on a un peu tendance à penser, a priori, que le personnage poétique c'est le personnage comme tu disais silhouetté, fugitif aussi, un peu flou. II n'a pas tout ce qu'a le personnage balzacien, il n'a pas son chapeau, sa grand-mère... Mais on a plus de peine à le penser comme un personnage qui fait: on le pense plutôt comme un personnage qui contemple, qui rêve... Or, Robinson, comme Plume d'une certaine façon, qui est au restaurant, qui prend le train, il passe son temps à faire des choses... C'est ça qui me paraît le plus intéressant, cette conjonction des deux aspects, cette dimension poétique qui n'est pas une sorte de déprise, du rêveur, joli... C'est un poétique dans l'action, dans un présent.

O.C. : C'est sans doute pourquoi j'ai écrit le plus souvent au présent. Pour écrire Le colonel, j'utilise ce qu'on appelle l'occupation de pensée dans le sport par exemple. C'est une didascalie vivante. Il commente en direct en agissant en virtuel ? Ou en vrai? C'est là où se pose ensuite la question du théâtre.

D.R. : Gardons cette idée compliquée qu'il y aurait un régime du personnage poétique. C'est une hypothèse : il y aurait un régime du personnage réaliste qui serait plutôt de l'ordre du constatif, il a une sorte d'existence supposée et il sert à décrire ; alors que le personnage poétique, version moderne, Michaux et toi, serait du côté d'une sorte de performatif, il accomplit.

O.C. : C'est ce que j'ai essayé de faire, je crois.

DR: Mais ça va plus loin, peut-être vers le roman, en tout cas vers une forme longue narrative... Car tu as besoin en même temps de montrer comment ça marche... Tu disais tout à l'heure: "ce n'est pas juste que je ne garde pas que le poème, ce n'est pas que du 
performatif", tu as besoin aussi d'une espèce d'enveloppe qui montre de façon comique et ironique parfois, comment ça se fabrique, ça s'emballe, ça dérape, ça s'arrête, ça rate...

o.C. : Tout ça me permet d'avoir des choses purement poétiques comme enchâssées. Et quasi invisibles. Pour que ça passe par un autre canal. Ce qui est curieux c'est que ne voulant pas user la méthode utilisée par mon premier livre et faire un nouveau livre de poésie, je l'ai converti en romanesque. J'ai construit ce personnage qui était au départ mon père - j'ai abandonné assez vite l'idée que c'était mon père, je l'ai appelé $\mathrm{R}$ d'abord (René). J'ai écrit “Enfance de $\mathrm{R}$ :". Je voulais me retrouver derrière lui, peut-être pour réhabiliter chez lui quelque chose de la tendresse, bien avant qu'il ne devienne réellement tendre à la fin de sa vie, et aimant. Mais sa souffrance, sa douleur, pour plein de raisons, étaient telles qu'il fallait que je réconcilie nos deux enfances dans un présent pur. Dans Le Colonel des Zouaves, on se déplace complètement dans ce présent, et ce personnage, je peux enfiler son costume. Je suis son cobaye, je peux me glisser dans sa peau et peut-être transfigurer mes propres obsessions. Il travaille pour moi, il est à mon service. Simplement après, il y a le théâtre qui arrive, et qui rend la chose encore plus complexe et plus simple. Car c'est Laurent Poitrenaux qui vient incarner ce Robinson, et qui l'incarne corporellement.

D.R. : Le fait que Poitrenaux incarne Robinson, qu'est-ce que ça fait, qu'est-ce que ça change?

0.C. : Un personnage est maintenant projeté sur scène en live donc, et au lieu de voir un personnage, un sujet X., on voit un livre ouvert. Poitrenaux, ce qu'il fait, c'est qu'il phrase le texte, au sens où il le déphase, il bouge et il fait le texte, donc c'est un personnage, bien sûr c'est un personnage, mais : au moment même où on va vouloir s'identifier à lui, rapport classique avec le personnage, on ne peut pas, parce qu'il n'a que deux mètres carrés de jeu. Il est pris dans une espèce de cage en fil de fer, qui fait qu'on a l'impression qu'il est derrière une vitre. Au fond c'est un papillon cloué dans une collection. C'est un livre en marche. Il y a un type qui entend des voix : qu'est-ce qu'on fait, on prend dix personnes pour le faire? Ou on ne prend qu'une seule personne? On ne prend qu'un seul personnage pour en faire quinze? Bien sûr il faut prendre un acteur alors... Et dans son Robinson, il y a deux personnages. Parce que le théâtre nous amène un personnage en réel et l'autre en virtuel, on pourrait dire. Sur scène, c'est à la fois un livre et une performance. Ça peut se jouer à l'IRCAM, aussi bien que dans des lieux de théâtre. Pour moi, cet étrange objet réconcilie la performance sonore avec le théâtre. On a le son prodigieux de Poitrenaux. On a le son et au lieu de faire une polyphonie, comme je disais, avec quinze acteurs, on construit un machine sonore démultipliée comme si c'était un roman qui se déroulait devant nos yeux ! Et puis il y a la danse. Odile Duboc a fait travailler Laurent Poitrenaux pour les passages poétiques, les courses à pied. Quel est le statut de cet art poétique-là qui consiste à courir et à voir des choses? Est-ce que la poésie n'est pas un afflux ? une montée ? ... D'ailleurs c'est une bonne expression au théâtre: quand un acteur se prend un peu trop au sérieux, on dit qu'il a une montée de personnage.

D.R. : Avant d'en venir (enfin) à Médecine générale, parlons de Providence.

O.C. : Oui, c'est la première épreuve avec la question du personnage. Dans le livre qui précède, Un mage en été, Robinson est mort, je l'ai tué, comme Conan Doyle avec Sherlock Holmes. Je n'ai plus de Robinson. Dans Providence le premier chapitre commence avec une espèce de rébellion du personnage contre son auteur... j'ai un peu honte de cette ficelle, six personnages en quête de n'importe quoi, c'est un peu 
facile, mais j'en avais besoin : j'avais besoin de mal me parler, "me" c'est-à-dire Robinson en moi, c'est-à-dire le Robinson lyrique justement, il fallait que je fasse taire cette voix qui commençait à devenir trop, facile, qui commençait à devenir virtuose, comme d'ailleurs je l'étais devenu un peu trop en lisant, c'est pour ça que je ne fais plus beaucoup de lectures publiques. Qu'est-ce qui se passe donc? Je fais Providence: il n'y a plus de narrateur central qui affronte un ennemi, mon père est mort. C'est ridicule, mais c'est ainsi, je me suis réveillé un matin en me disant : je ne pourrai plus faire de fiction. Et je l'ai dit, ce qui est bizarre, à haute voix. En plus le mot fiction, je ne l'emploie jamais !

D.R. : C'est un mot d'époque!

o.c. : C'est un mot que je ne comprends pas, mais bref je l'ai quand même dit, verbalisé, comme le mot d'époque : je n'ai plus besoin de faire de fiction. Quand j'étais petit et que j'étais pénible, mon père me punissait quelquefois violemment et parce qu'il se sentait très coupable ensuite, il venait me voir dans ma chambre et me disait : maintenant tu vas pouvoir te créer un personnage. C'est-à-dire: tu vas pouvoir faire la victime, vas-y.

D.R. : Plains-toi?

0.C. : Le jour du procès, tu pourras t'inventer un personnage. Voilà notre problème $\mathrm{du}$ jour qui pointe son nez. Il ajoutait, pour rire: n'oublie pas ce qu'on fait aux parricides, on leur coupe la main et on la plonge dans du plomb fondu. Voilà, c'était le genre de phrase...

D.R. : Pas de fiction... mais après tu en fais quatre dans Providence.

o.C. : C'est ça, j'en fais quatre. Robinson s'est diffracté. Je n'ai pas de solution donc je prends quatre avatars séparés. Qu'est-ce qu'il se passe après? Je me retrouve devant le chantier de Médecine générale, et là ça se complique. Je veux rassembler trois personnages dans la même action. Il ne faut pas avoir inventé la poudre pour appeler ça roman.

D.R. : Une trinité ?

0.C. : Oui, une Trinité... Et donc qu'est-ce que je fais ? Je fais une première version du livre, et dans ce premier manuscrit, je raconte un certain nombre de choses qui vont m'arriver exactement, après : avec une chronologie exacte, le héros part aux ÉtatsUnis, comme moi, après, je pars aux États-Unis comme si le livre était devenu programmatique. Les trois héros se retirent à la campagne, je fais de même. Quand le héros entend des voix dans Futur, ancien, fugitif, vingt ans plus tard j'en entends en vrai, mais là, c'est en six mois que ça se passe, le temps entre la prédiction et sa réalisation est très court. J'ai presque eu peur. Donc j'ai abandonné la publication pour prendre une année de travail en plus, j'ai fait une bonne petite crise, je suis parti sous la pluie, et je me suis tapé quatre mois de boulot l'hiver... Juste avant le confinement, bonne idée ! Et qu'est-ce que je fais? Je travaille sur de grandes feuilles. Il n'y a rien au centre, il y a un vide. Et il y a un personnage qui s'appelle Mathilde : je commence à faire presque comme dans un jeu vidéo où on présente un personnage et on prend ses points de vie (ou ses points de mort). Pour la première fois, j'ai eu un très grand plaisir en faisant ces placards, $j$ 'en ai fait beaucoup. Je deviens un formateur de personnages. Et j'arrive, par chance énorme au bout de quatre mois, à étoffer suffisamment ces petites saynètes de la première version du manuscrit et à faire que les personnages sortent du monologue. 
D.R. : Médecine générale m'apparaît comme un bouclage et comme une manière de relancer tout le cycle... La question se pose de savoir s'ils sont bien trois dans le roman, si c'en est un... À la fin ce n'est pas Providence qui recommence, ils viennent lui dire : "tu nous saoules. D'abord tu n'es pas malade, et puis on est autre chose que ce que tu as voulu qu'on soit". Et donc une fin plutôt heureuse. Est-ce qu'il faut dire que ton héros consent à l'altérité ? Qu'il laisse Mathilde et Pierre exister? Pierre qui me semble une sorte de double de Laurent Poitrenaux.

O.C. : Bien sûr, c'est l'innocent.

D.R. : C'est celui qui apprend à toute vitesse...

O.C. : Bien sûr, et c'est un grand acteur, c'est ce que disent les deux autres de lui. C'est un personnage à trois têtes aussi. Pour la première fois, je ne suis pas en mono. Je ne suis pas en mono, je suis en trilogue grâce à eux. Parce qu'ils ont suffisamment de force, les autres, pour pouvoir rentrer dans le truc. Médecine générale c'est la naissance d'un nouveau Robinson - mais bien entouré.

D.R. : Oui, parce qu'il a les mêmes obsessions que Robinson: repartir à zéro, rien comprendre, refonder un monde, aménager... il garde quelque chose de très Robinson, mais avec Mathilde il a une sœur d'adoption, et une partie de son projet est devenu celui de sa sœur, ce qui est quand même très différent quant aux archives familiales...

o.C. : C'est ça! Avec Mathilde et Pierre, je veux le beurre et l'argent du beurre. Je veux que ce soit exactement ça, parce que c'est ça qui m'excite, je veux habiller le schéma de chair et de sang.

N.P. : Tu travailles en ce moment à un nouveau projet.

O.C. : Oui, je suis devant un nouveau texte, à faire pour Christoph Marthaler, le metteur en scène. Christoph n'aime pas le théâtre... chez lui tout ce qui est de l'ordre du texte est toujours ridicule... Tout ce qui est émotif, charnel et sensuel passe par la musique. Donc ça va être assez compliqué de faire un texte pour quelqu'un qui n'en veut pas, selon l'algorithme amoureux de Lacan... Et je suis pris, là, de manière très simple, par un désir de réalisme, c'est-à-dire de proposer vraiment de faire une pièce de théâtre, avec des personnages pour le coup... La seule contrainte : il y a trois acteurs, deux actrices qui parlent beaucoup et un troisième qui ne parle pas.

D.R. : Deux femmes, un homme?

O.C. : Deux femmes, un homme, j'ai essayé plein de solutions, d'actions. Je voulais placer l'histoire dans un restaurant, vraiment un truc réaliste, une arrière-salle de restaurant où lui n'arrête pas d'astiquer le bar et de faire monter et descendre un monte-charge qui s'ouvre tout le temps [bruitage], et les deux femmes qui épluchent des patates et qui en fait s'avèrent être des philosophes de choc. J'ai abandonné cette option, ce serait un pastiche des pièces de Marthaler. J'ai abandonné les personnages et je me dis que je vais travailler par fragments, puisqu'après tout Christoph travaille comme ça. Il faut que je trouve la ou les voix qui peuvent déployer ses fragments.

N.P. : Donc c'est toujours la question de la forme du livre qui se pose.

O.C. : Oui, toujours. Mais pas dans l'idée d'un livre objet. C'est une question de volume, et de surgissement de la voix dans l'espace typographique du livre. J'ai donc quelquefois peur de me tromper. C'est-à-dire d'avoir créé moi-même un système d'enchevêtrement théorico-pété excessif, et je ne peux pas m'asseoir dessus, en profiter et savoir ce que je veux à l'avance. Je ne peux pas m'en servir. Je suis obligé de repartir à zéro à chaque fois que je fais un livre. À chaque fois il y a une dialectique qui doit se résoudre dans une sorte de grâce. Il faut une désinvolture aussi pour le 
faire... Ce n'est pas un effort théorique, c'est un effort purement corporel, je dirais que c'est un rapport au corps. Donc c'est du corps, et c'est mon corps qui est en jeu. Alors là on abandonne, on termine notre histoire de personnage en fait : je fais ce que je peux. Corporellement, pour aller loin, parce qu'en fait je bouge beaucoup en écrivant, je marche, j'ai besoin d'espace, j'ai besoin de circuler, je n'écris pas au sens classique, je fais toujours plus ou moins ça [montre le schéma personnage griffonné plus tôt]. Je fais des recherches, quelquefois il y a quelque chose qui s'écrit au centre et qui peut ressembler par chance extraordinaire, comme le voyage de mon frère dans mon dernier livre, à une vraie prose. Une vraie prose que tu as eue comme ça, flaf! Et après c'est comme une folie pour que tout ça fasse des ondes circulaires, que tout s'aimante.s

\section{AUTEURS}

\section{NATHALIE PIÉGAY}

Université de Genève

DOMINIQUE RABATÉ

Université de Paris 